\title{
Linx
}

Revue des linguistes de l'université Paris X Nanterre

$72 \mid 2015$

Former à l'écrit universitaire, un terrain pour la linguistique?

\section{Gérer la modalité épistémique et le hedging : analyse comparée d'examens écrits en Lettres et en Sciences humaines}

\section{Thierry Herman}

\section{OpenEdition}

\section{Journals}

Édition électronique

URL : http://journals.openedition.org/linx/1641

DOI : $10.4000 /$ linx.1641

ISSN : 2118-9692

\section{Éditeur}

Presses universitaires de Paris Nanterre

Édition imprimée

Date de publication : 1 septembre 2015

Pagination : 151-168

ISSN : 0246-8743

\section{Référence électronique}

Thierry Herman, «Gérer la modalité épistémique et le hedging : analyse comparée d'examens écrits en Lettres et en Sciences humaines », Linx [En ligne], 72 | 2015, mis en ligne le 01 mars 2016, consulté le 01 mai 2019. URL : http://journals.openedition.org/linx/1641; DOI : 10.4000/linx.1641 


\title{
Gérer la modalité épistémique et le hedging : analyse comparée d'examens écrits en Lettres et en Sciences humaines
}

\author{
Thierry HERMAN \\ Universités de Neuchâtel et de Lausanne, Suisse
}

\section{Résumé}

Cet article présente une exploration préliminaire à une recherche portant sur un macro-genre particulier: l'examen écrit de 3è année de Bachelor en lettres et en sciences humaines (l'équivalent de la licence en France). En comparant un corpus de bons examens et d'examens ratés, nous avons cherché à illustrer des différences dans la gestion de la modalité épistémique et du hedging, à savoir «des moyens linguistiques utilisés pour indiquer soit 1) un manque d'engagement complet envers la valeur de vérité exprimée dans la proposition qui l'accompagne soit 2) le désir de ne pas exprimer cet engagement de manière catégorique » (Hyland 1998: 1). Nous avons tenté de comprendre si des examens jugés insuffisants pratiquent une modalité épistémique très catégorique alors que des travaux jugés excellents font varier plus explicitement les degrés d'engagement par rapport aux propositions énoncées. Il apparaît que ce sont moins les hedges que les boosters et la force de certitude qui marquent une différence entre bons et mauvais examens.

\section{Mots-clés}

Hedges, boosters, modalité épistémique, examen écrit, certitude.

L'idée à la source d'article est fondée sinon sur un paradoxe, du moins sur une tension, entre une situation de communication ou une situation rhétorique (Bitzer, 1968), qui est celle de l'étudiant se présentant à un examen écrit, et la conduite du texte académique destiné à être évalué ${ }^{1}$. En effet, l'étudiant rédigeant un écrit pour un évaluateur expert se situe dans un rapport de places (Flahault, 1979) qui lui assigne par essence une position inférieure sur le plan du savoir et des compétences par rapport à l'examinateur, alors que, dans l'écrit, c'est lui qui doit guider le lecteur et faire croire à une certaine assurance en prenant position sur la vérité des propositions. Au cœur de l'examen règne donc la tension analysée par Reuter en 2004, tension qui implique «la recherche d'un équilibre délicat entre les positions de chercheur et de formé (d'apprenti-chercheur) entraînant soit l'oscillation entre une position trop basse et une position trop haute, soit une dérive consistant en la suraccentuation de l'une ou de l'autre de ces postures » (2004: 23).

Cette tension ou cette contradiction peut sans doute être étudiée dans différents lieux, mais l'un d'eux paraît être la question de la gestion de la modalité épistémique. Cette dernière, dans la définition de Coates (1987 : 112), indique, «dans la plupart des cas, la confiance ou le manque de confiance que le locuteur a dans la vérité des propositions qu'il asserte ». Associer cette idée de confiance au cadre anxiogène des examens finaux de Bachelor paraît bien cristalliser l'enjeu de cette question linguistique. D'autant plus que ce qui a alimenté la réflexion à la source de cet article est une observation intuitive paradoxale selon laquelle les meilleurs travaux d'examens émanaient d'étudiants dont la force d'engagement dans les dires était mesurée, sinon prudente. Autrement dit, les assertions très catégoriques formulées par certains étudiants semblaient recevoir, de manière générale, moins de crédit que des écrits pratiquant ce que Lakoff a nommé le hedging, qui permet de rendre les choses plus ou moins

\footnotetext{
${ }^{1}$ Je remercie les relecteurs anonymes pour leurs remarques sur la première version de ce document.
} 
floues («make things more or less fuzzy »). L'enjeu de cette contribution est donc d'observer si cette intuition était fondée.

Pour ce faire, j'ai opté pour une démarche d'analyse comparée d'examens de Bachelor en Lettres et en Sciences humaines en prenant un échantillon de 10 examens jugés très bons (note de 5 à 6 - note maximale en Suisse) et de 10 examens insuffisants (notes inférieures à 4). Ces examens proviennent de différentes disciplines enseignées dans la Faculté des Lettres et Sciences humaines de l'Université de Neuchâtel : philosophie, psychologie et éducation, sociologie, ethnologie, français, histoire, information et communication, géographie. Nous n'avons pas fait d'observation en fonction de l'ancrage disciplinaire - la taille du corpus ne permettant pas ce type de comparaison ${ }^{2}$. Il va de soi que l'enjeu est bien d'enqueter sur une possible corrélation entre travaux jugés bons et présence de hedging, mais pas de prouver une éventuelle causalité entre gestion de la modalité épistémique et appréciation de la note : il ne s'agit pas de prétendre que la gestion du hedging détermine le résultat de l'évaluation, mais tout au plus de signaler que c'est peut-être l'un des facteurs possibles.

\section{Hedging, écrit scientifique et genre de l'examen écrit}

La question du hedging formulée par Lakoff a une trajectoire particulière dans son histoire. Pour Hyland, en 1998, les définitions sont «étonnement rares » ${ }^{3}(1998: 1)$ et parfois surmontées d'une couche idéologique faisant apparaître l'idée de tromperie ou de manipulation. Depuis son étude sur cette notion dans les articles de recherches scientifiques (1998), de nombreux pans de recherche en linguistique sont entrés en corrélation pour aboutir à une réflexion sur la question de la certitude, par le biais des notions de subjectivité, de modalité épistémique ou encore d'évidentialité (Rubin, Liddy et Kando, 2005). La définition souvent citée des hedges - nous garderons ici cette appellation plutôt que les « enclosures » de la traduction proposée par Kleiber et Riegel (1978) - est celle de Ken Hyland : «tout moyen linguistique utilisé pour indiquer soit 1) un manque d'engagement complet envers la valeur de vérité exprimée dans la proposition qui l'accompagne soit 2) le désir de ne pas exprimer cet engagement de manière catégorique » (Hyland, $1998: 1$ ); en cela, ils s'opposent aux «boosters » 4 et font partie intégrante de différentes catégories du métadiscours proposées par Hyland (2005). Depuis, de nombreux travaux se sont focalisés sur la question de la modalité épistémique et de la certitude. Nous mentionnerons en particulier plusieurs travaux de Rubin (Rubin et al., 2005 ; Rubin, 2007), laquelle propose un modèle de catégorisation de la certitude que l'on peut appliquer au corpus. Cette question des degrés de certitude inscrite dans les textes est analysée de manière catégorielle par Hyland, qui repère ainsi des formes lexico-grammaticales et des stratégies pour mettre en scène des hedges dans un texte. Je ne suivrai pas ici cette volonté taxinomique, mes travaux s'inscrivant plutôt dans une démarche rhétorique (cf. par exemple Herman, 2009) réfléchissant aux effets possibles des hedges ou de leur absence.

En tant qu'analyste de discours, je suis particulièrement attaché à la question des spécificités génériques des textes. Or, le genre de l'examen écrit en sciences humaines et sociales est peu décrit dans la littérature scientifique : dans la liste que propose Fanny Rinck dans son état des

\footnotetext{
${ }^{2}$ La taille réduite du corpus s'explique par le fait qu'il s'agit ici d'une recherche préliminaire en vue de déposer un projet de recherche en 2013. Les résultats présentés le sont à titre indicatif.

${ }^{3}$ A des fins de lisibilité, je propose dans cet article une traduction des travaux en anglais.

${ }^{4}$ La première condition renvoie à des aspects énonciatifs et sémantiques alors que la seconde s'appuie sur la dimension pragmatique des hedges. J'ai choisi pour cette première enquête de ne pas distinguer entre les deux fonctions, la taille du corpus ne permettant pas de montrer des différences significatives.
} 
lieux en 2010, je ne trouve pas trace de ce genre de discours et n'ai vu quant à moi qu'un article de Marie-Christine Pollet (2004) sur ce type de corpus. Je ne soulèverai ici que quelques points méritant une investigation plus fine. Les contraintes situationnelles sont connues : il s'agit de répondre, en un temps restreint, à une question donnée ${ }^{5}$, réponse qui sera évaluée par un lecteur expert jugeant de l'étendue des connaissances et des savoir-faire acquis durant l'enseignement dispensé. La nécessité de démontrer un savoir acquis implique de fait que la gestion de la modalité épistémique paraît constituer un enjeu crucial du genre. Cette nécessité de développer un ethos de sachant pour un lecteur expert montre que le hedging peut apparaittre comme contre-productif dans ce genre particulier. On pourrait du coup observer dans le corpus une tendance à la surassertion (Maingueneau 2004) - ou à la manifestation d'un haut degré de certitude de peur que tout affaiblissement volontaire du propos marque un manque d'assurance rédhibitoire.

Une autre caractéristique forte de l'examen réside dans l'importance de la définition. En effet, ce genre implique souvent, en sciences humaines et sociales, la mention d'une ou de plusieurs notions clés dans l'intitulé de la question ${ }^{6}$, dont il est attendu une définition ou des explications. Or, là aussi, on retrouve une pression du côté de la gestion épistémique : le micro-genre de la définition renvoie intuitivement à des ouvrages de type dictionnaire ; autrement dit, la référence à un texte péremptoire, relativement définitif, paraît être cognitivement très présente. Il sera dès lors intéressant de voir dans le corpus les essais de définitions et leur parenté avec une définition de type dictionnaire impliquant un effacement énonciatif (Rabatel) selon lequel tout hedging peut être perçu comme une anomalie.

Enfin, une troisième caractéristique propre à de nombreux examens - je ne saurais dire à ce stade de mes recherches leur proportion quantitative - mobilise certes des notions, mais souvent rattachées à des auteurs. Et donc, une des dimensions importantes de l'examen écrit consiste à gérer la parole d'autrui, activité problématique pour les étudiants (cf. Boch et Grossmann, 2001). Or, une nouvelle fois, l'effacement énonciatif du scripteur de l'examen constitue une conséquence directe de l'activité résumante qui doit élaborer un discours saturé par des marques d'évidentialité et par des citations.

En somme, ces trois caractéristiques génériques, que l'on retrouve partiellement dans d'autres écrits académiques, mais qui paraissent assez exacerbées dans le cas des examens écrits, poussent à l'effacement énonciatif. Mais comme le souligne Rinck à propos de l'impersonnel, cela «ne signifie pas une absence de positionnement et au contraire même, il peut servir des assertions fortes » (2010: 440). En effet, l'effacement énonciatif se combine, sur le plan des modalités, à un engagement très fort sur la certitude des propos tenus : que ce soit en résumant la pensée d'un auteur, en définissant des notions-clés ou, plus largement, en faisant

\footnotetext{
${ }^{5}$ Il s'agit dans ce corpus d'examens de fin de bachelor ( $3^{\mathrm{e}}$ année universitaire) portant sur un ou plusieurs cours disciplinaires dans lesquels on demande au candidat de répondre à une ou deux questions qui demandent des développements. Voici quelques exemples à titre d'illustration: «Dans quelle mesure peut-on parler de la culture comme d'une "seconde nature" »; "Comment est-ce que l'anthropologie distingue les explications de type matérialiste des explications de type symbolique ? Illustrez votre réponse avec des exemples tirés des textes de Bodley. Ensuite, montrez les limites de cette distinction à travers ces exemples ou d'autres»; «La socialisation politique : une forme parmi d'autres de socialisation »; « Après avoir expliqué la différence entre connaissance a priori et connaissance a posteriori, ainsi que la différence entre propositions analytiques et synthétiques, expliquez le problème rencontré par Kant à propos d'une certaine catégorique de jugements qui ne sont ni analytiques, ni a posteriori. Enfin, dites pourquoi ce problème était important aux yeux de Kant » ...

${ }^{6}$ Encore faut-il distinguer ici des sous-genres de l'examen : il est vrai que des commentaires composés, par exemple, échappent à une telle nécessité. Dans le cadre de cette recherche, nous n'avons pas mis en relation les types de questions posées et les sous-genres de l'examen écrit avec les phénomènes linguistiques - hedges et boosters - observés. Ce sera une dimension intéressante de notre future recherche.
} 
preuve de son savoir, l'étudiant-e en examen se voit contraint-e de mobiliser avec force un engagement dans la certitude de ce qu'il avance, puisque c'est précisément ce sur quoi il va être évalué.

Le problème est que l'article scientifique s'inscrit dans un genre qui se démarque sur ce plan. On peut même dire l'activité scientifique entière fait de même. Pour Ken Hyland par exemple, les hedges «sont abondants en science et jouent un rôle crucial dans l'écrit académique. Les hedges sont importants pour les scientifiques parce que, [...] même les propositions scientifiques les plus assurées ont par essence un temps d'acceptation limité » (1998:6). Il cite un peu plus loin Crismore et Farnsworth pour qui les hedges sont «la marque du scientifique professionnel » $(990: 135)$ et montre que les hedges sont les marques métadiscursives les plus fréquentes de l'écrit scientifique (2005). Entre en jeu dans cette conception la question de la rhétorique scientifique: la nécessité de convaincre autrui se marque par une recherche de consensus qui passe par l'expression des doutes et de la prudence. «En d'autres termes, les hedges sont un outil rhétorique important dans l'écrit académique dans la mesure où ils signalent que le locuteur anticipe les objections de ses propositions » et qu'ils peuvent « aider à garantir l'acceptation du lecteur » (1998: 7). Hyland touche ainsi un point important à la fois par rapport à l'activité et à la rhétorique scientifiques.

Mais peut-on transférer ces points, liés de manière cruciale aux articles scientifiques, au genre de l'examen écrit? Si l'activité scientifique et ses normes sont transférables, la situation rhétorique, elle, est différente : dans un examen, il ne s'agit pas de convaincre à propos d'un contenu inédit, mais de convaincre sur le plan de l'ethos, autrement dit, de répondre à des attentes en termes de savoir et de compétences qui justifient la réussite ou non de l'examen. Les étudiants pourraient être tentés de vouloir d'abord se montrer sûrs d'eux-mêmes, en percevant cette posture comme un avantage dans l'évaluation des compétences, sans prendre garde que, ce faisant, ils donneraient une image d'eux-mêmes bien différente de celle que l'on peut lire en tant que chercheurs dans des articles scientifiques. On peut dès lors se demander quelle grille de lecture adoptent les personnes qui évaluent : celle qu'ils appliquent à un article scientifique ou celle qu'ils utilisent pour le genre particulier de l'examen ? On peut supposer que le risque de confusion existe et que l'absence de hedging soit comprise comme une faiblesse dans les compétences.

Cela nous permet d'aborder la question de la modalité épistémique et de la certitude. En 2005, Rubin, Liddy et Kando proposent d'élaborer une grille d'analyse de la certitude sur quatre plans que Rubin raffine en 2007 pour aboutir à ce schéma : 


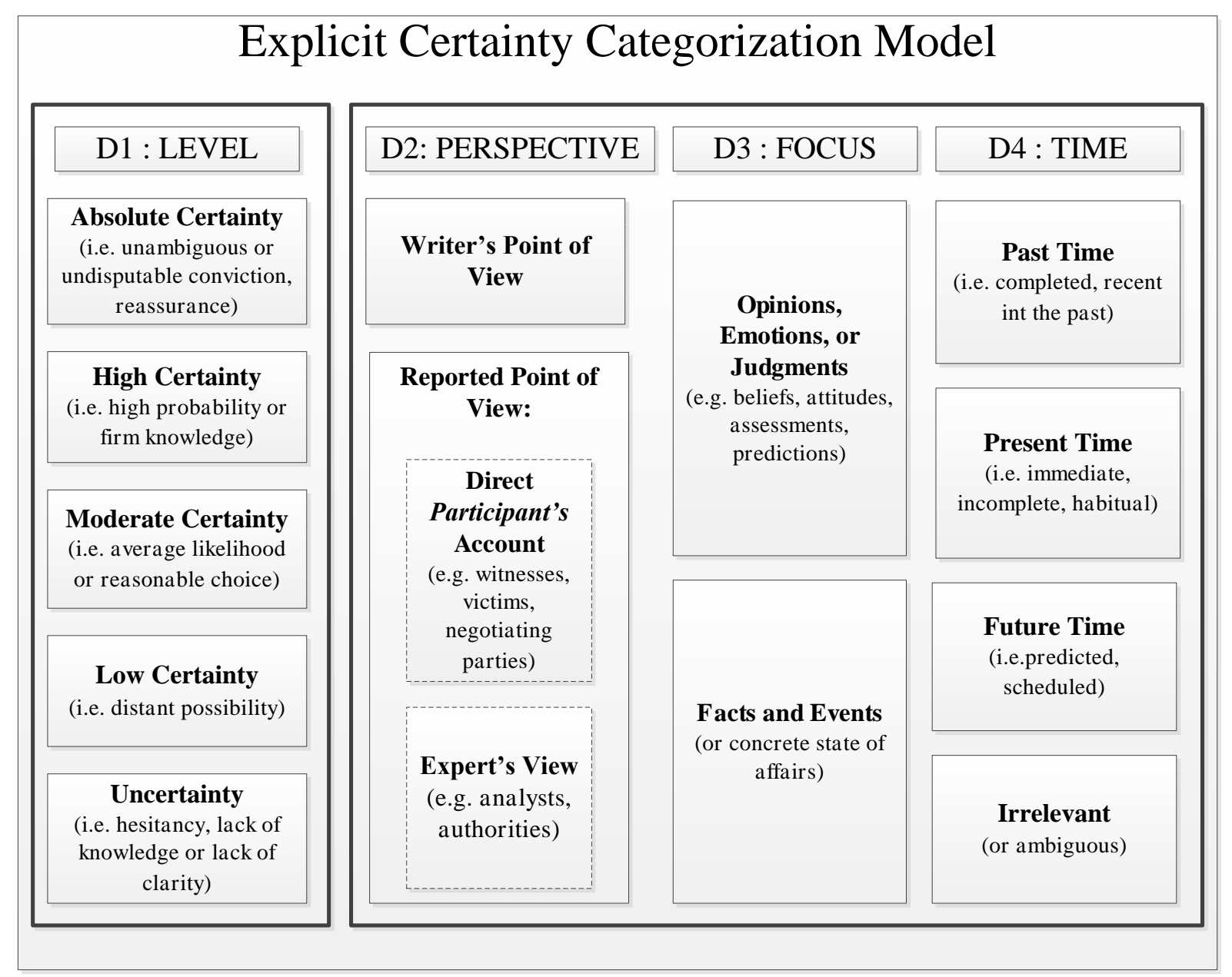

Si ce schéma est d'un grand intérêt, il souffre d'un problème qui est la volonté de classer sur l'échelle de certitude des marques explicites (Rubin, 2007 : 142), considérant par exemple que «sans aucun doute» marque une certitude quasi absolue alors que «sans doute » une certitude faible. Ainsi, Rubin ne tient pas compte des propositions non-marquées. Or, on le verra lors de l'analyse de corpus, les propositions non-marquées sont largement dominantes. De même, l'intéressante troisième colonne proposant de distinguer la certitude portée sur les opinions et celle sur les faits risque d'écraser toute forme de différence entre modalité aléthique et épistémique. C'est pourquoi il faut associer à ce schéma l'importante et dense réflexion de Laurent Gosselin (2010) sur les modalités en français.

Ce dernier distingue, dans son «analytique des modalités », différents paramètres parmi lesquels l'instance de validation, la force de validation, le degré d'engagement du locuteur. L'instance de validation fait écho à la proposition de Berrendonner (1981) distinguant les agents vérificateurs que sont les locuteurs, l'opinion commune ou l'univers référentiel - le «fantôme de la vérité ». Gosselin distingue pour sa part trois instances de validation. La première est la réalité, qui présente la fois une stabilité et une fiabilité maximale: «La validité du jugement n'est pas soumise aux variations subjectives. C'est en quoi elle peut être dite «objective », elle ne dépend que de la « réalité » qu'elle exprime (exemples : la terre est ronde, il neige, etc.).» (2010: 67). Il s'agit en ce cas de la modalité aléthique. Deuxième instance : la validité subjective, qui se caractérise à l'inverse par sa variabilité et «par la fiabilité minimale des informations communiquées» (ibid.). Enfin, entre les deux, «une région dans laquelle les jugements, quoique non objectifs, sont en quelque sorte stabilisés, car dépendants de systèmes de conventions visant à une certaine généralité » (ibid.) : il s'agit 
d'une validité conventionnelle ou institutionnelle. Gosselin montre encore qu'il ne faut pas assimiler le subjectif et l'individuel. Ainsi «La soupe est bonne » est bien subjectif mais se livre comme une appréciation collective alors que «je trouve que la soupe est bonne » est présenté comme individuel. Il nous semble intéressant de relever ce double point, car il fait écho à des enjeux centraux de l'écrit de recherches : le tabou de l'expression individuelle qui est déjà bien documenté - et la pression de dire la réalité. On peut ainsi imaginer que des opinions individuelles subjectives se dissimulent sous une voix collective et que des contenus proprement épistémiques s'affirment avec le poids de l'aléthique.

Deuxième paramètre intéressant dans le cadre de l'analyse : la force de la validation. C'est ce paramètre qui permet de faire varier les degrés de certitude d'une modalité épistémique. Gosselin propose un continuum entre l'exclu et le certain, avec une série de degrés de certitude ou d'incertitude. Enfin, le troisième paramètre concerne la force de l'engagement ou la prise en charge énonciative. Gosselin affirme qu'il est nécessaire de dissocier force d'engagement et degré de croyance à l'égard de la proposition exprimée. Ainsi « je t'assure qu'il risque de pleuvoir » combine un engagement maximal sans pour autant se présenter comme certain. Il est à noter que l'assertion sans marque explicite, «Marie est malade » est considérée par Gosselin comme une prise en charge très forte du locuteur, même si elle peut être encore renforcée par « Je t'assure que Marie est malade » ou «Je te jure sur la tête de mes enfants que Marie est malade »(Gosselin, 2010 : 226).

Les points résumés ici sont facilement confondus : l'échelle de certitude de Rubin ne tient pas compte de la force d'engagement du locuteur ni de l'instance de validation. En outre, le caractère subjectif assumé ou non par une expression individuelle n'est pas analysé non plus. En ce sens, les outils que donne Gosselin paraissent précieux pour mesurer certains phénomènes liés aux modalités aléthiques ou épistémiques et d'autres, liés à l'assertion.

\section{Analyse du corpus}

\subsection{Les hedges}

Livrons tout de suite un résultat peu spectaculaire. L'hypothèse d'une corrélation entre présences de hedges ${ }^{7}$ et évaluation globale du travail formulée en introduction est quantitativement plutôt confirmée, mais sans plus. Concrètement, dans le corpus de 10 bons examens écrits, je dénombre six travaux mettant en place 21 occurrences de hedges ${ }^{8}$, parmi lesquels :

(1) L'enquête de terrain, qui repose entre autres sur la pratique de l'observation participante, se trouve au cœur de la démarche ethnologique.

(2) En effet, il nous semble qu'un point essentiel de la problématique soulevée concerne des imprécisions définitionnelles

(3) Les concepts clés de cette approche sont notamment

(4) On peut distinguer des approches de type matérialistes [ $\left.\mathrm{sic}^{9}\right]$

(5) La tentative de donner une définition acceptable de ce qu'est une connaissance est de toute évidence l'une des plus lourdes tâches de la philosophie épistémique

\footnotetext{
${ }^{7}$ Il faut préciser que n'ont été prises en considération ici que des formes lexicalisées de hedging.

${ }^{8}$ Précisons que nous avons considéré que le début de chaque examen - environ une page et demie de texte - tant pour des raisons techniques que pour équilibrer les résultats.

${ }^{9}$ Pour toutes les citations, l'orthographe d'origine est conservée.
} 
Adverbes ou locutions adverbiales $(1,3)$, verbes modaux $(2,4)$, nom (5) ou adjectif (5) sont ainsi essentiellement utilisés à des fins de préservation de l'ethos du locuteur. Les limites des hedges se portent essentiellement sur deux aspects : le premier vise l'atténuation d'un référent qui serait trop général $(1,3)$ - par prudence, on restreint la portée générale de l'énoncé en jouant sur le critère de force de la validation; le second vise à amoindrir la force de l'initiative que prend le locuteur $(2,4,5)$ : on retrouve visiblement ici une forme d'ethos de modestie classique. L'exemple (2) en particulier montre un cas peu fréquent d'instance de validation subjective assumée comme telle par une présentation individuelle, même si le «nous » indique une pseudo-collectivité. Dans les autres cas, les instances de validation semblent être de l'ordre de l'institution ou de la doxa - l'usage du « on » collectif dans (4) en lieu et place d'une première personne qui paraît tout aussi acceptable donne un surplus d'autorité au propos, faisant écho ou feignant de le faire à des divisions épistémologiques pratiquées par des experts. Il n'en demeure pas moins que, pour une stratégie considérée comme classique de la rhétorique scientifique, le nombre de hedges paraît singulièrement peu élevé. Constater que quatre travaux sur 10 n'offrent dans l'échantillon choisi aucun hedge lexicalisé paraît très troublant.

Du côté du corpus des mauvais examens, 7 travaux sur 10 mentionnent des hedges pour treize occurrences. Certes, le nombre d'occurrences en contenant est plus faible, mais ce chiffre est si peu élevé que la différence ne me parait pas très révélatrice. En voici quelques exemples :

(6) On pourrait comparer cela aux idées émises par Piaget

(7) Il est peu probable que les préférences politiques de l'enfant s'éloignent considérablement de celles des parents

(8) Ce travail aura pour but d'essayer de répondre à la question suivante

On trouve dans ces exemples des caractéristiques similaires à celles observées auparavant : ethos de modestie (8), proposition atténuée par un modal (6) ou une tournure épistémique (7).

Au final, le plus surprenant dans cette récolte de hedges est donc sa maigreur, eu égard à cette «marque du scientifique professionnel ». On retrouve, sans doute de manière exacerbée dans l'examen écrit, ce que Delcambre et Reuter constataient dans le rapport à l'écriture de recherche qu'entretiennent les étudiants : «doute et modalisations sont tendanciellement évacués de leurs écrits par les étudiants, non en raison d'un rapport au savoir qui leur serait propre ou d'une position à laquelle ils « adhéreraient », mais en fonction d'images des textes réflexifs » (2002: 24). Introduisons ici une nuance dans le cas des examens écrits : l'image des textes réflexifs ne paraît pas jouer un si grand rôle. En effet, si on suit Hyland, l'écrit de recherche, même en sciences dures, est truffé de hedges. Pour expliquer ce paradoxe, je suis amené à lancer trois hypothèses : la première est que les étudiants narcotisent cette présence en se faisant leur image de l'écrit de recherche lors de leurs lectures, s'attachant peut-être prioritairement aux résultats concrets qu'aux doutes, nuances, hésitations et prudences ; la seconde est que l'image est faussée ou dévoyée par une autre pratique qui est l'enseignement universitaire : celui-ci, visant l'apprentissage de notions, est-il peut-être plus définitif, moins nuancé que les écrits scientifiques - surtout, il s'inscrit dans la suite de l'enseignement préuniversitaire. L'idée que l'on puisse mettre en doute les propositions et les notions enseignées n'est sans doute pas naturelle; la troisième est que la situation même de l'examen écrit imprègne plus directement les réalisations énonciatives des étudiants qui se sentent obligés d'exhiber un savoir présenté avec une certitude absolue. J'ai tendance à penser que la troisième exerce un poids considérable, résultant de la tension mentionnée en début d'article. L'avantage que présente cette hypothèse explicative est que l'on peut l'affermir par des 
indices linguistiques du corpus.

\subsection{Boosters}

Il paraît étonnant que Hyland ait consacré une monographie sur les hedges qui mentionne l'importance des marqueurs d'emphase (boosters) en laissant de côté cependant l'observation parallèle de ce phénomène, alors qu'il montre que mélanger les deux est intéressant. Ainsi, les exemples 2 et 5 montrent que les atténuations des hedges contrebalancent la force des boosters au sein du même énoncé, cumulant les avantages de la force sémantique des derniers et de la prudence énonciative des premiers. En outre, la surprise des travaux d'examens est le nombre plutôt élevé de marques d'emphase (21 occurrences dans le corpus de bons examens et 36 dans les mauvaises copies). On trouve ce genre d'exemples :

(9) Il est indispensable de préciser qu'afin que la transmission ait lieu, deux conditions sine qua non (Correcteur souligne et met en marge un ?) doivent exister

(10) Je dois encore préciser qu'il est devenu aujourd'hui très difficile de parler de préférences politiques tant celles-ci sont devenues variées et vastes.

(11) Une fois ces questions répondues, il faut forcément se demander ce qu'est l'inflation.

(12) Donc les modèles culturels (et du coup, les symboles) sont très importants (souligné en ondulé) pour l'individu.

(13) Pour pouvoir passer de la socialisation 1ère à la socialisation secondaire la seule façon c'est la conversion religieuse (phrase soulignée et, en marge, Non ! Pas du tout !)

(14) Ce concept fondamental de notre question est très controversé.

(15) Pour comprendre ce qu'est l'impératif catégorique, il faut inclure différentes notions telles que devoir, volonté, règles morales, raison.

Tout d'abord, il faut savoir qu'un impératif est une règle.

Au-delà de la diversité des formes de boosters, les exemples livrent plusieurs enseignements intéressants. Premièrement, une forme d'inconscience du risque pris lors du choix d'une marque d'emphase. Le nombre de boosters trouvés sans encadrement par un atténuateur, en particulier dans les travaux insuffisants, semble signaler une volonté affichée de se montrer sûr de soi et catégorique dans l'affirmation au point d'accentuer la force de validation au-delà de ce qui est nécessaire ou de ce que la prudence commanderait. À cet égard, dans le corpus des bons examens, on ne trouve que peu d'occurrences de boosters qui ne soient pas contrebalancés par des atténuateurs dans le même énoncé (voir exemples 2 et 5). Dire ainsi « il nous semble très important », qui combine la volonté de mettre en relief et l'atténuation par un avis strictement individuel, semble une stratégie mieux acquise par les bons travaux, alors que les mauvais travaux feront, à l'instar de (9), des affirmations unilatéralement fortes. Deuxièmement, on peut le voir dans les exemples $9,10,12$ et 13, les marques d'emphase sont souvent rejetées par les correcteurs des examens. C'est d'autant plus notable que, de manière générale, on trouve assez peu de remarques de corrections dans ce corpus - certains se contentent d'une remarque finale avant de mettre une note. On voit bien ici une forme de résistance par rapport à une stratégie linguistique qui apparait sortir du prototype générique ou ne pas respecter les valeurs scientifiques de prudence et circonspection. On trouve ainsi un exemple de correction proposant l'insertion de ce que Charolles (1997) appelle un cadre de discours pour atténuer la portée générale du propos : 
(16) (en marge : traditionnellement, ...)Les sciences humaines s'intéressent et traitent de l'humain dans ses attitudes, ses convictions, ses habitudes et ses croyances.

On voit dans ce dernier exemple, une assertion non modalisée, posée comme évidente, dont la certitude épistémique dérange l'évaluateur qui demande une atténuation du propos, une réduction à un cadre traditionnel. Cela incite à observer le cas de figure le plus fréquent : la phrase sans hedge ni booster.

\subsection{Certitude}

Ce qui surprend dans l'analyse du corpus est le nombre d'assertions non modalisées, et ce, que ce soit dans les bons ou dans les mauvais travaux. La faible présence de hedges explicites est évidemment un indice en faveur d'un tel constat. Voici le premier paragraphe d'un examen qui exacerbe une tendance qui paraît assez générale :

(17) $\mathrm{Au}$ début du 19e siècle, apparaît le travail à domicile. Les entrepreneurs veulent tirer profit de la main-d'œuvre «transparente » : les femmes et les enfants. Ce système est très avantageux pour l'entrepreneur. Mais à la fin du $19 \mathrm{e}$ siècle, les visions changent. On valorise plus le travail en usine que le travail à domicile. Cela a été possible grâce à des innovations dans plusieurs secteurs industriels, à savoir le secteur textile, sidérurgique et enfin le secteur des transports. On commence à s'industrialiser.

On notera dans cet exemple l'absence de marques de transition explicites à l'exception de «mais », le peu de justifications données aux différents points mentionnés, l'absence de toute référence à des sources primaires ou secondaires - toutes formes d'indices métadiscursifs que Hyland range avec les hedges et les boosters dans son modèle du métadiscours. Chaque énoncé est posé comme une évidence, comme l'indiquent de nombreux présents de vérité générale. La nature avant tout épistémique des énoncés convoqués est ainsi dissimulée au profit d'une apparence aléthique. Pour Gosselin, rappelons-le, la modalité épistémique présente des « "vérités subjectives" : des jugements encore essentiellement descriptifs, qui ne constituent pas des "jugements de valeur", et qui pourtant ne renvoient pas à une réalité indépendante des sujets qui la considèrent, mais à l'évaluation subjective de cette réalité » (2010 : 325). La modalité aléthique concerne, elle, «la vérité objective » où les jugements sont aussi descriptifs mais «renvoient à une réalité existant en soi, indépendants des jugements qui sont portés sur elle»(2010: 314). L'effacement de la subjectivité des jugements dans ce qu'elle a d'individuel par des assertions de vérité générale non modalisées, non prises en charge et alignées dans un moule parataxique conduit à faire passer des jugements de vérité subjective comme des jugements de vérité objective ${ }^{10}$. On le voit dans l'exemple suivant, où la relative présuppose un accord sur une définition de la psychologie sur laquelle l'évaluateur réagit par des remarques sceptiques et une demande de citation confirmatoire :

(18) Le fait d'apprendre est au cour de la psychologie qui est la science de l'âme (remarque : qui dit cela ? Et le corps ? Aucun rôle ?). C'est dans notre tête que se passe l'action d'apprendre et d'acquérir un savoir.

\footnotetext{
${ }^{10}$ Cette suite d'affirmations catégoriques aurait évidemment présenté un autre visage avec l'usage de métadiscursifs, des hedges en particulier.
} 
C'est prendre un risque considérable dans un examen écrit dans le cas où, précisément, l'affirmation non factuelle est discutable. Tout se passe comme si la dimension argumentative de l'écrit était occultée au profit d'une dimension descriptive : on raconte ce que l'on sait plutôt que de convaincre d'un point de vue. Cela provoque un des dysfonctionnements repérés par Reuter lorsqu'il évoque des modalités énonciatives inadaptées : " assertions sous forme de vérités générales, prescriptions (il faut/on doit/ il est nécessaire de...), axiologisation marquée, enchaînements non étayés (les enseignants font... les élèves apprennent) » (2004 : 13).

Dans ce tableau général, on peut distinguer néanmoins deux situations qui parfois d'ailleurs s'entrecoupent : celle des séquences de définitions et celle des paroles tierces rapportées. Les exemples (19), (20), (21) et (22) illustrent ceci. Les exemples (19) et (20) sont une séquence de pure définition des termes, l'exemple (21) une définition selon un auteur ou une définition rapportée, l'exemple (22), des propos rapportés

(19) En résumé, on parle ici de la différence entre ontogenèse et phylogenèse. L'ontogenèse est donc la vie « d'esprit » d'un individu, c'est-à-dire son développement personnel dans un environnement donné, pendant que la phylogenèse décrit le parcours génétique avec la recherche et la compréhension des propriétés génétiques des humains et de leurs ancêtres (double ondulé en marge).

(20) [...] apprendre c'est trouver sa place dans la société, faire en sorte qu'elle soit reconnue par les autres puis savoir utiliser les outils qui sont mis à disposition afin d'atteindre ses buts et d'évoluer.

(21) Une proposition (ou jugement, chez Kant) analytique est une proposition dont le concept du prédicat est inclu dans le concept du sujet, selon la définition de Kant. Une proposition synthétique est une proposition dont le concept du prédicat n'est pas inclu dans le concept du sujet, toujours selon Kant.

(22) Sa conception de l'Etat est un Etat fort, symbolisé par le Léviathan. L'Etat est tout-puissant, il n'y a rien en-dessus de lui. La paix est maintenue grâce à la peur. En effet, la peur de représailles suffit à empêcher les conflits.

Hobbes dit que pour observer la nature humaine, il faut dépouiller l'homme de tout ce qui différencie des autres (culture, langue, habit, etc.). En faisant cela, on observe l'homme dans ce que Hobbes appelle l'Etat de nature.

On retrouve dans les quatre cas des assertions très générales présentées avec un haut degré de certitude. Dans les deux premiers exemples, on peut suspecter la pression de la norme du dictionnaire qui pousse à présenter les concepts et leurs différences intrinsèques de manière définitive. Notons une fois encore que cette force affirmative est rejetée par l'examinateur dans le cas 19, qui accole au paragraphe des traits ondulés signalant le caractère très approximatif de la définition. Les exemples 21 et 22 émanent de travaux qui ont reçu une très bonne note et qui font abriter une série d'assertions très catégoriques sous l'égide d'un auteur : Kant ou Hobbes. En ce sens, le travail de résumé d'une pensée ou de définition d'une notion, en changeant son instance de validation ou sa perspective (colonne 2 du schéma de Rubin), est moins frontalement pris en charge par le locuteur. On peut se demander toutefois, vu la multiplication des assertions catégoriques, si les étudiants ont conscience de la différence; comme l'affirment Boch et Grossmann : "Que ce soit dans les synthèses de textes, dans les dissertations ou dans des écrits plus longs tels que les rapports de stage, la polyphonie n'est pas maîtrisée : les voix sont souvent brouillées et le correcteur a bien du mal, parfois, à démêler la voix de l'étudiant de celle des auteurs qu'il convoque; bref, de façon 
récurrente, on ne sait pas "qui parle" » (2001 : 91). À cet égard, la différence est très nette dans le corpus entre les bons travaux et les mauvais : alors que, on le voit dans l'exemple 21, les bons n'hésitent pas à rappeler parfois avec insistance que le propos n'est pas le leur, mais celui d'un auteur convoqué par un " selon », les mauvais travaux brouillent souvent l'instance de validation. En comparant le corpus sous cet angle, la différence quantitative est saillante. Dans le corpus de bons examens, même si on repère que certains examens, dans les premières lignes choisies, ne font mention d'aucune source externe, on découvre une vingtaine d'occurrences de propos rapportés en «selon » ou indexés par un cadre de discours détaché en tête d'énoncé du type "dans son ouvrage ». Dans le corpus de mauvais examens, en revanche, on ne trouve que 5 occurrences dans trois travaux. Si on accepte la remarque de Reuter selon laquelle «le discours théorique (et/ou de recherche) me parait être fondamentalement - un discours d'autrui » (2001 : 14), on mesure bien que les étudiants qui ratent leur examen ne semblent pas avoir intégré cette dimension et préfèrent se réapproprier le savoir qu'ils doivent livrer, assumant ainsi une prise en charge des propos qui dépasse parfois leurs compétences ou leurs savoirs.

À cet égard, le tabou que représente la présence du locuteur dans le texte scientifique ne simplifie pas le rapport aux données. Sans surprise, on ne trouve que quatre occurrences du pronom «je » dans du méta-discours organisationnel : «je vais commencer par», «je vais prendre l'exemple de », «je dois préciser », « je pourrai faire une distinction ». La présence des nous d'auteur est plus importante, surtout dans le corpus des bons travaux. On ne trouve que trois travaux qui mentionnent le nous d'auteur dans le corpus des mauvais examens dominé par des impersonnels et le pronom «on » comparé à sept travaux dans les bons examens. Rappelons qu'il s'agit d'un corpus de premières pages d'examens, plutôt propices à un positionnement de l'auteur. Ici, je renvoie aux travaux de Fløttum et al. (2007), de Hyland (2002) ou encore de Tutin (2010) sur l'observation de cette gestion des pronoms dans les articles de recherche. Ces travaux montrent en effet un fort effacement énonciatif, variables selon les disciplines et selon les cultures scientifiques, sans pour autant, et cela paraît être une différence cruciale avec le corpus des étudiants en formation, renoncer à une dimension rhétorique qui est, pour Tutin, fortement présente $(2010, \S 1)$.

\section{Conclusion}

Bien qu'il soit difficile de tirer des conclusions définitives sur la base de ce corpus et bien qu'il faille sans aucun doute compléter nos observations sur des textes complets, ce premier panorama confirme partiellement notre hypothèse de départ, mais met surtout en évidence que la contrainte des examens écrits semble limiter l'apparition même des hedges. Lorsqu'ils apparaissent, ces derniers proposent surtout un ethos conventionnel, quasi ritualisé, qui est un ethos de modestie. En regard de la présence massive des boosters et de la certitude absolue affichée dans une très nette majorité d'énoncés, la faible présence des hedges semble attester qu'une des normes de l'examen telle qu'elle est vue par les scripteurs est de manifester une très forte assurance. L'idée de doser la modalité épistémique en faisant varier les degrés de certitude ou même de montrer une forme d'incertitude ne semble pas spontanée. D'une certaine manière, ce qui semble dominer n'est plus du tout la modalité épistémique, mais une modalité aléthique forcée dans laquelle s'opère un transfert de prise en charge de l'énonciateur : ce dernier affirme des vérités en son nom propre plutôt que de les déléguer à une autorité de tutelle dont il se fait pourtant une sorte d'écho; par là même, il fait une reconstruction personnelle qui est parfois, souligne Marie-Christine Pollet avec un certain sens du booster, « à cent mille lieues du sens initial » (2004 : 83). 
Le cumul d'assertions catégoriques crée un effet de linéarisation du contenu : on ne voit pas vraiment de hiérarchie entre les affirmations qui se juxtaposent. En somme, cette observation confirme le constat de Pollet: "Différentes données sont présentées successivement, indépendamment l'une de l'autre, comme si elles de participaient pas à un ensemble organisé » (2004: 83). Cette dernière renvoie à un travail de Boch et Frier qui évoquent «le besoin de sécurité, le faible coût en traitement cognitif et la volonté de mise à distance ». Mais cette logique de stockage paraît moins saillante sur le plan des transitions - on voit quand même apparaître plusieurs connecteurs dans les deux corpus choisis - que sur le plan de la modalité énonciative où les étudiants livrent des données comme on livre un paquet, sans implication, sans présence, dans un souci de transparence qui correspond sans doute à une forme de représentation tronquée, faussée, de l'écrit scientifique neutre. À ce titre, la troisième colonne du schéma de Rubin, la distinction de la certitude portant sur les faits et sur les opinions, mériterait d'être approfondie dans le corpus des examens. Dans une première approche, il me semble que l'aspect linéaire et juxtaposé des assertions manifestant un haut degré de certitude conduit à ne pas différencier la nature des données manipulées.

Au final, la dimension rhétorique de l'écrit scientifique n'apparait que comme faiblement intégrée en fin de leur troisième année d'études universitaires alors même que les étudiants qui semblent la mâtriser le mieux sont aussi ceux qui produisent des examens jugés bons à très bons. C'est d'ailleurs pourquoi mon enseignement, tourné sur le rôle de la rhétorique dans l'écrit académique, appuie à plusieurs reprises sur l'importance de guider le lecteur et donc de marquer une présence, de faire comprendre ce que l'on fait autant que ce que l'on dit dans une sorte de pseudo-dialogue avec le lecteur et non d'une simple livraison de données. Ce premier panorama d'un corpus en voie de constitution m'incite à donner plus de place au niveau de mon enseignement à la gestion des hedges et des boosters comme des traces de présence discursive contribuant à l'ethos de scripteur/locuteur pour tenter de sortir de cette logique du stockage décrite par plusieurs auteurs.

\section{Bilbiographie}

BERRENDONNER, A, 1981, Eléments de pragmatique linguistique, Paris, Minuit. BITZER, L., 1968, « Rhetorical situation », Philosophy and Rhetoric, 1, p. 1-14.

BOCH, F., GROSSMANN, F. (dir.), 2001, « De l'usage des citations dans le discours théorique : des constats aux propositions didactiques », Lidil, 24, p. 91-112.

CHAROLLES, M., 1997, «L'encadrement du discours : univers, champs, domaines et espaces », Cahier de recherche linguistique, 6, p. 1-73.

COATES, J., 1987, «Epistemic modality and spoken discourse », Transactions of the Philological society, 85, p. 100-131.

DELCAMBRE, I., REUTER, Y., 2002, « Images du scripteur et rapport à l'écriture », Pratiques, $121 / 122$, p. 7-28.

FLAHAULT, F., 1979, La parole intermédiaire, Paris, Seuil.

FLØTTUM, K., T., DAHL, T., KINN, T., 2007, Academic Voices, Amsterdam, John Benjamins.

GOSSELIN, L., 2010, Les modalités en français, La validation des représentations, Amsterdam, New York, Rodopi.

HERMAN, T., 2009, «Rhétorique des incipit dans les articles scientifiques en sciences humaines et sociales », dans J.-M. Defays (dir.), Les discours universitaires : formes, pratiques, mutations, Paris, L'Harmattan.

HYLAND, K., 1998, Hedging in Scientific Research Articles, Amsterdam, John Benjamins. HYLAND, K., 2002, "Authority and Invisibility : authorial identity in academic writing », 
Journal of Pragmatics, 34, p. 1091-1112.

HYLAND, K., 2005, « Metadiscourse : Mapping Interactions in Academic Writing », Nordic Journal of English Studies, 9(2), p. 125-143.

Kleiber, G., Riegel, M., 1978, «Les grammaires floues », dans R. Martin (dir.), La notion de recevabilité en linguistique. Paris, Klincksieck, p. 67-124.

LAKOFF, R., 1972, « Hedges : a study in meaning criteria and the logic of fuzzy concepts », CLS, 8, p. 183-228.

MAINGUENEAU, D., 2004, «Citation et surassertion », Polifonia, Cuiabà (Brésil), 8, p. 122.

POLLET, M.-C., 2004, « Appropriation et écriture des savoirs chez des étudiants de première année- Une voie difficile entre stockage et élaboration », Pratiques, 121-122, p. 81-92.

RABATEL, A., 1998, La construction textuelle du point de vue, Paris-Lausanne, Delachaux \& Niestlé.

REUTER, Y., 2004, «Analyser les problèmes de l'écriture de recherche en formation », Pratiques, 121-122, p. 9-27.

REUTER, Y., 2001, «Je suis comme un autrui qui doute », Lidil, 24, p. 13-27.

RINCK, F., 2010, «L'analyse linguistique des enjeux de connaissance dans le discours scientifique », Revue d'anthropologie des connaissances, 4(3), p. 427-450.

RUBIN, V.L., Liddy, E., Kando, N., 2005, « Certainty Identification in Texts : Categorization Model and Manuel Tagging Results », dans J.G. Shanahan, Y. Qu, J. Wiebe (dir.), Computing Attitude and Affect in Text : Theory and Applications, New York, Springer.

RUBIN, V.L., 2007, «Stating with Certainty or Stating with Doubt : Intecoder Reliability Results for Manual Annotation of Epistemically Modalized Statements », Proceedings of NAACL HLT, companion volume, p. 141-144.

TUTIN, A., 2010, "Dans cet article, nous souhaitons montrer que... Lexique verbal et positionnement de l'auteur dans les articles en sciences humaines », Lidil, 41, p. 15-40. 